\title{
Potential for application of an acoustic camera in particle tracking velocimetry
}

\author{
Fu-Chun Wu, ${ }^{1}$ Yun-Chuan Shao, ${ }^{1}$ Chi-Kuei Wang, ${ }^{2}$ and Jim Liou ${ }^{3}$ \\ ${ }^{1}$ National Taiwan University, Taipei 106, Taiwan \\ ${ }^{2}$ National Cheng Kung University, Tainan 701, Taiwan \\ ${ }^{3}$ University of Idaho, Moscow, Idaho 83844, USA
}

(Received 15 June 2008; accepted 17 October 2008; published online 17 November 2008)

\begin{abstract}
We explored the potential and limitations for applying an acoustic camera as the imaging instrument of particle tracking velocimetry. The strength of the acoustic camera is its usability in low-visibility environments where conventional optical cameras are ineffective, while its applicability is limited by lower temporal and spatial resolutions. We conducted a series of experiments in which acoustic and optical cameras were used to simultaneously image the rotational motion of tracer particles, allowing for a comparison of the acoustic- and optical-based velocities. The results reveal that the greater fluctuations associated with the acoustic-based velocities are primarily attributed to the lower temporal resolution. The positive and negative biases induced by the lower spatial resolution are balanced, with the positive ones greater in magnitude but the negative ones greater in quantity. These biases reduce with the increase in the mean particle velocity and approach minimum as the mean velocity exceeds the threshold value that can be sensed by the acoustic camera. (C) 2008 American Institute of Physics. [DOI: 10.1063/1.3021046]
\end{abstract}

Particle tracking velocimetry (PTV) is now a well established and frequently used technique of experimental fluid mechanics. ${ }^{1,2}$ By seeding the flow with tracer particles and registering particle displacements over short time intervals, information on the fluid motion can be acquired. The optical cameras employed in typical PTV allow for the fast acquisition of high-resolution digital images, often in a wellilluminated laboratory setting. In the field, acquisition of target images frequently has to be performed in underwater environments with poor visibility, where conventional optical cameras are ineffective. Acoustic cameras have emerged as a good alternative for obtaining images of underwater objects and have been used as real-time monitoring devices in turbid water. ${ }^{3,4}$

To date, however, no such effort has been made to use acoustic cameras as the imaging tool for PTV in lowvisibility environments. Here we explore the potential for applying an acoustic camera as the imaging sensor of PTV. A series of laboratory experiments is conducted in which acoustic and optical cameras are simultaneously used to track two-dimensional (2D) particle motion, allowing for a comparison of the acoustic- and optical-based velocities. The discrepancies induced by the differences in the temporal and spatial resolutions of the acoustic and optical cameras are demonstrated and analyzed.

Experiments were conducted in a pump sump channel located at the sub-basement of the Hydraulics Laboratory, University of Idaho. The channel was $13 \mathrm{~m}(\mathrm{~L})$ by $1.5 \mathrm{~m}(\mathrm{~W})$ by $2.4 \mathrm{~m}(\mathrm{H})$ and filled with water $1.2 \mathrm{~m}$ in depth. At the end a dual-frequency identification sonar (DIDSON) acoustic camera (Sound Metrics Corp., Lake Forest Park, WA) was set up at $0.6 \mathrm{~m}$ from the bottom and tilted $10.1^{\circ}$ upward to image the observation area at the water surface (Fig. 1). Over the observation area a Sony charge coupled device (CCD) digital video camera was installed on a cross-channel rod $0.85 \mathrm{~m}$ above the water surface. Five $8 \mathrm{~mm}$ plastic beads (numbered 1-5) were hung from a $30 \mathrm{~cm}$ horizontal bar and driven by a variable-speed motor to make circular motion at the water surface. Such a setting was to assure that particle motion was strictly in a 2D configuration with no vertical motion involved. All beads were partially submerged and thus simultaneously seen by the two cameras.

A total of nine runs were performed, with the rotation speeds ranging from 3.1 to $14.5 \mathrm{rpm}$. In each run, rotation of tracer particles was maintained for $60 \mathrm{~s}$. The video sequences of particle motion were acquired using both cameras. The acoustic camera operated at 10 frames/s. The 96 $\times 512$ pixel polar-coordinate images (averagely 12.8 $\times 2.5 \mathrm{~mm}^{2} /$ pixel) were internally mapped to the Cartesian coordinates, with an output resolution of $505 \times 560$ pixels $\left(2.5 \times 2.1 \mathrm{~mm}^{2} /\right.$ pixel $)$. The CCD camera operated at 30 frames/s, with an image resolution of $720 \times 480$ pixels $\left(0.85 \times 1 \mathrm{~mm}^{2} / \mathrm{pixel}\right)$. After all runs, a calibration grid board was placed on the observation area and imaged by both cameras. The calibration images were used to establish the relations between the image and physical coordinates.

The procedure for image processing includes (1) noise removal, (2) particle positioning, ${ }^{5-7}$ and (3) geometric calibration. ${ }^{8}$ Particle trajectories can be traced from a series of images, and the velocities can be estimated.

The rotation trajectories of four tracer particles (beads 1-5) for runs 5 and 6 are demonstrated in Fig. 2. The two runs were, respectively, the maximum and minimum rotation speeds used in this study. The results obtained by the two cameras are in satisfactory agreement. The acoustic-based trajectories, however, exhibit a more sinuous pattern than the optical-based ones. The frame rate of the optical camera is three times faster than that of the acoustic camera. Moreover, the resolution in the near-range optical image is much finer than the mean resolution in the far-range acoustic polarcoordinate image. With such differences in both the temporal and spatial resolutions, the trajectories extracted from the 




FIG. 1. Overall view of the experimental setup. Rotation of tracer particles is driven by a variable-speed motor. Particle motion is simultaneously imaged by a down-looking CCD camera and an up-looking DIDSON acoustic camera.

acoustic images are inherently less smooth than those extracted from the optical ones. These acoustic-based trajectories in turn will lead to more fluctuating velocities.

Shown in Figs. 3(a) and 3(b) are the acoustic- and optical-based instantaneous particle velocities along the phase angle of circular trajectories (clockwise from the negative $x$ axis). The acoustic-based velocities exhibit greater fluctuations than the optical-based ones. The mean velocities and fluctuations of four tracer particles (run 5) are summarized in Table I. Three discrepancies between the acousticand optical-based velocities are observed. (1) The acousticbased mean velocities are consistently greater than the corresponding optical-based ones (averagely 3.8\% greater). (2) The acoustic-based mean fluctuations are consistently greater than the corresponding optical-based ones (averagely 2.7 times greater). (3) The relative mean fluctuations of the optical-based velocities remain as low as $\sim 5 \%$, while those of the acoustic-based velocities are two to three times greater, particularly greater are those with lower speeds. The consistently greater mean values and fluctuations of the acoustic-based velocities are attributed to the lower temporal and spatial resolutions.

To explore the effects of temporal and spatial resolutions, we propose to downgrade the temporal resolution of
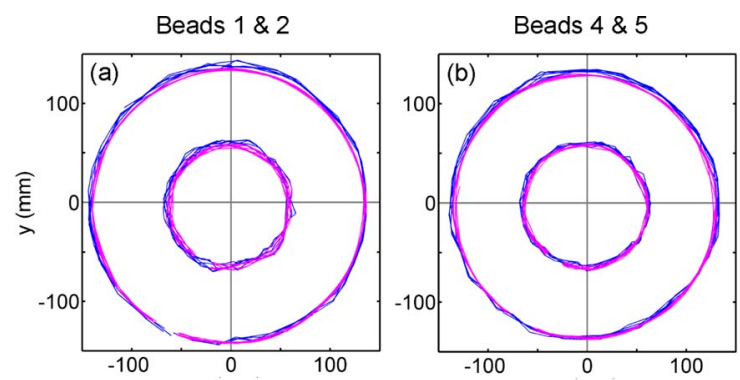

$14.5 \mathrm{rpm}$
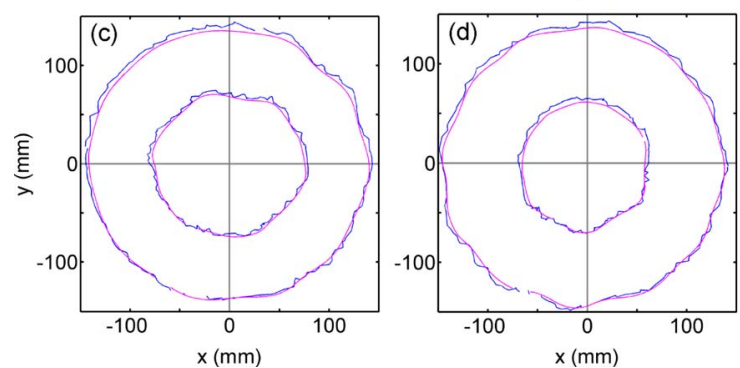

$3.1 \mathrm{rpm}$

FIG. 2. (Color online) Rotation trajectories of beads 1 and 2 (left panels) and beads 4 and 5 (right panels): [(a) and (b)] run 5, and [(c) and (d)] run 6 . Red lines are results extracted from optical images; blue lines are results extracted from acoustic images.
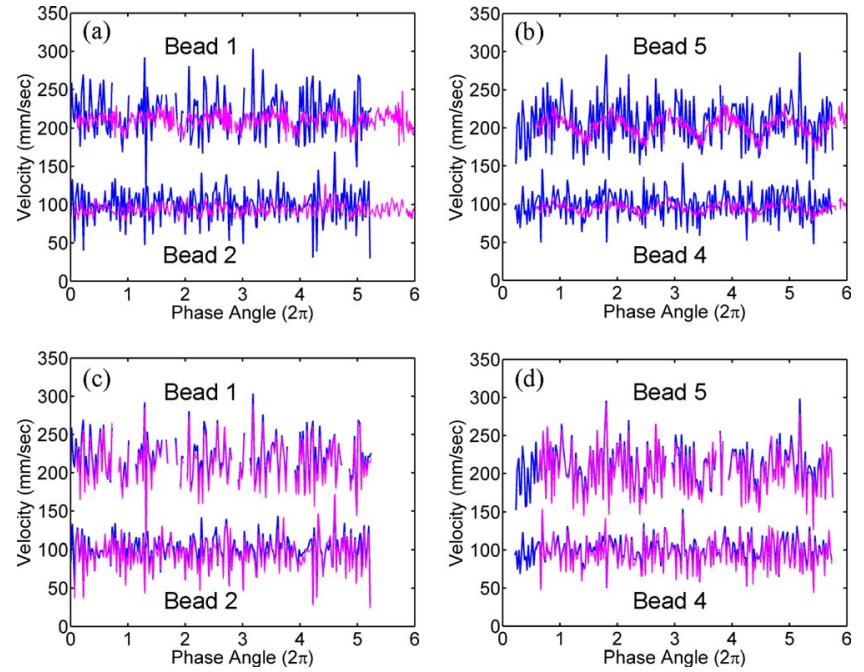

FIG. 3. (Color online) [(a) and (b)] Acoustic- and optical-based instantaneous velocities of four tracer particles along the phase angle of the circular trajectories. [(c) and (d)] Downgraded optical-based instantaneous velocities compared to the acoustic-based instantaneous velocities. Blue and red lines are the acoustic- and optical-based results, respectively (data from run 5).

optical images. A comparison between the original and downgraded optical-based velocities would reveal the effect of temporal resolution; the effect of spatial resolution could be separated from the combined effect if the optical and acoustic images had an identical temporal resolution. This is done by interpolating particle positions on the optical-based trajectories such that the phase angles of the interpolated positions would be fully consistent with those on the acoustic-based trajectories. The downgraded optical-based instantaneous velocities are then obtained with the interpolated particle positions, as shown in Figs. 3(c) and 3(d), where better agreement between the two types of velocities is observed. The mean values and fluctuations of the downgraded optical-based velocities are also summarized in Table I. The mean fluctuations of the downgraded optical-based velocities are nearly identical to the corresponding acousticbased values, implying that greater fluctuations are primarily attributed to the lower frame rate. With the temporal resolution downgraded by a factor of 3 , the mean fluctuations

TABLE I. Comparison of acoustic-based, original, and downgraded opticalbased mean velocities and fluctuations of four tracer particles (run 5).

\begin{tabular}{|c|c|c|c|c|}
\hline & \multicolumn{4}{|c|}{ Bead No. } \\
\hline & 1 & 2 & 4 & 5 \\
\hline \multicolumn{5}{|c|}{ Acoustic based $\left(10\right.$ frames $\left./ \mathrm{s}, 2.5 \times 2.1 \mathrm{~mm}^{2} / \mathrm{pixel}\right)$} \\
\hline Mean velocity $\bar{V}_{A}(\mathrm{~mm} / \mathrm{s})$ & 215.5 & 98.0 & 97.8 & 205.2 \\
\hline Mean fluctuation $\bar{v}_{A}(\mathrm{~mm} / \mathrm{s})$ & 22.6 & 16.5 & 14.7 & 24.4 \\
\hline Relative mean fluctuation $\bar{v}_{A} / \bar{V}_{A}(\%)$ & 11 & 17 & 15 & 12 \\
\hline \multicolumn{5}{|c|}{ Optical based (30 frames $/ \mathrm{s}, 0.85 \times 1 \mathrm{~mm}^{2} /$ pixel) } \\
\hline Mean velocity $\bar{U}_{O}(\mathrm{~mm} / \mathrm{s})$ & 208.3 & 92.9 & 94.3 & 200.4 \\
\hline Mean fluctuation $\bar{u}_{O}(\mathrm{~mm} / \mathrm{s})$ & 8.6 & 5.9 & 4.8 & 10.5 \\
\hline Relative mean fluctuation $\bar{u}_{O} / \bar{U}_{O}(\%)$ & 4 & 6 & 5 & 5 \\
\hline \multicolumn{5}{|c|}{ Downgraded optical based $\left(10\right.$ frames $/ \mathrm{s}, 0.85 \times 1 \mathrm{~mm}^{2} /$ pixel $)$} \\
\hline Mean velocity $\bar{V}_{O}(\mathrm{~mm} / \mathrm{s})$ & 210.3 & 93.0 & 94.6 & 201.1 \\
\hline Mean fluctuation $\bar{v}_{O}(\mathrm{~mm} / \mathrm{s})$ & 22.7 & 16.2 & 15.6 & 24.3 \\
\hline Relative mean fluctuation $\bar{v}_{O} / \bar{V}_{O}(\%)$ & 11 & 17 & 16 & 12 \\
\hline
\end{tabular}




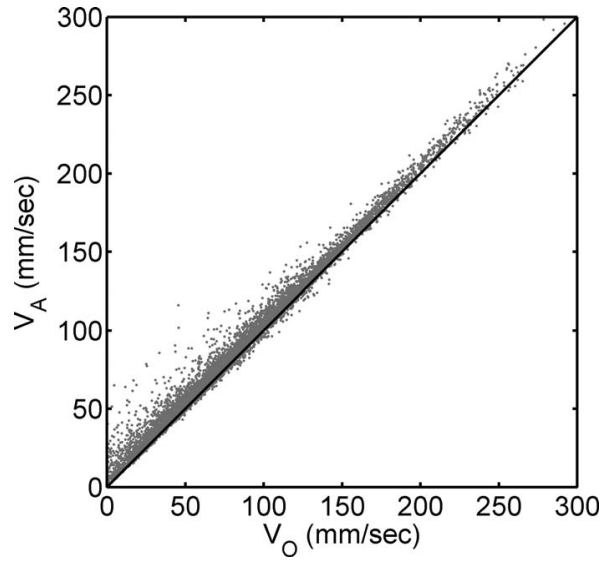

FIG. 4. Comparison between acoustic-based instantaneous velocities $\left(V_{A}\right)$ and downgraded optical-based instantaneous velocities $\left(V_{O}\right)$ of four tracer particles.

increased by an average of 2.7 times compared to the corresponding original optical-based values.

By contrast, the downgraded optical-based mean velocities become only slightly greater than the corresponding original values, while remain to be smaller than the corresponding acoustic-based values. A comparison of the acoustic-based instantaneous velocities $\left(V_{A}\right)$ and downgraded optical-based instantaneous velocities $\left(V_{O}\right)$ is shown in Fig. 4 , where the values of $V_{A}$ exhibit a strong tendency to exceed the corresponding values of $V_{O}$. Because the acoustic-based and downgraded optical-based results are of a temporal resolution, it is speculated that the discrepancy between the acoustic-based and downgraded optical-based velocities is due to the difference in spatial resolution.

To quantify the biases induced by the effect of spatial resolution, the acoustic-based velocities $V_{A}$ and downgraded optical-based velocities $V_{O}$ of each tracer particle are normalized with the corresponding mean velocities $\bar{V}_{A}$ and $\bar{V}_{O}$. The difference between the normalized velocities, $V_{A} / \bar{V}_{A}$ $-V_{O} / \bar{V}_{O}$, is then used as a quantitative measure of the bias induced by the difference in the spatial resolutions of the two cameras.

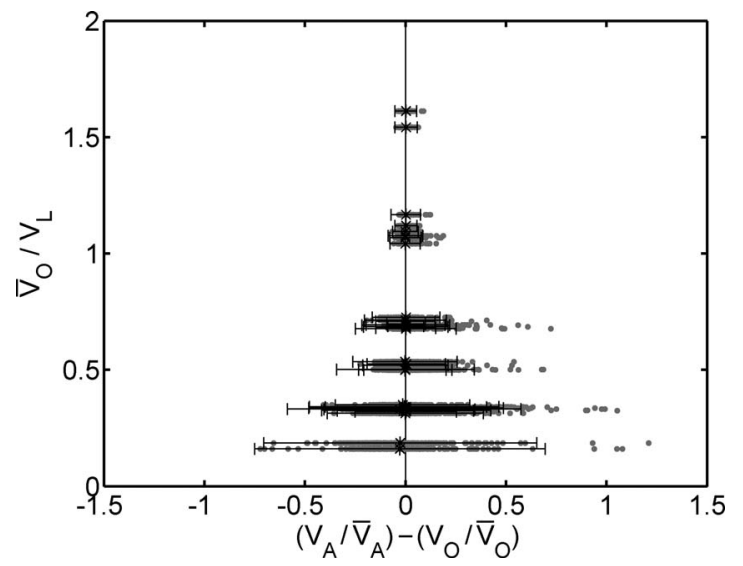

FIG. 5. The biases induced by the effect of spatial resolution, defined as $V_{A} / \bar{V}_{A}-V_{O} / \bar{V}_{O}$, varying with nondimensionalized mean particle velocity $\bar{V}_{O} / V_{L}$. A total of 36 data sets are shown; the mean value of each data set is marked with an asterisk, and the associated error bar represents the mean \pm three times of standard deviation.

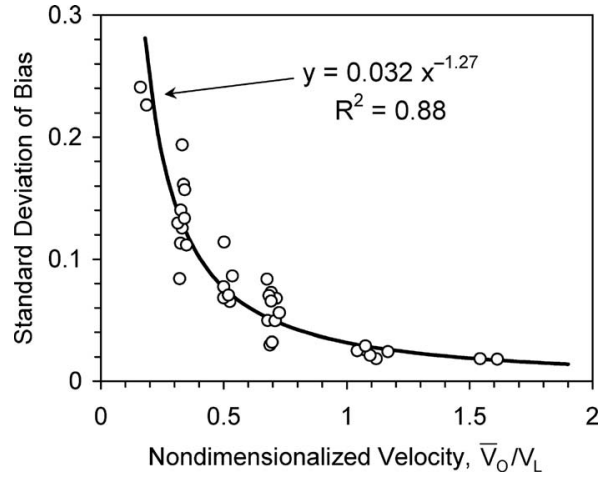

FIG. 6. Relation between the standard deviation of biases and nondimensionalized mean particle velocity.

The values of $V_{A} / \bar{V}_{A}-V_{O} / \bar{V}_{O}$ are shown in Fig. 5 for a range of $\bar{V}_{O} / V_{L}$, where $\bar{V}_{O}$ represents the mean particle velocity, and $V_{L}$ is the threshold particle velocity, defined as the lowest velocity that can be sensed by the acoustic camera. The value of $V_{L}$ is taken to be $130 \mathrm{~mm} / \mathrm{s}$, evaluated by considering a tracer particle that passes the diagonal of a mean pixel in the observation area over a time interval. A tracer particle with its instantaneous velocity less than $V_{L}$ could be mistaken as static. In all, 36 sets of biases are shown in Fig. 5 , where the biases due to the effect of spatial resolution can be positive or negative. The biases range from -0.8 to 1.3 for the smallest values of $\bar{V}_{O} / V_{L}$. The range of the biases reduces with the increase in $\bar{V}_{O} / V_{L}$ and approaches minimum as $\bar{V}_{O} / V_{L}$ exceeds unity.

The mean value of each set of biases is marked with an asterisk (Fig. 5), and the associated error bar represents the range of mean \pm three times of standard deviation. The mean biases are consistently close to zero, indicating that the total quantities of positive and negative biases are balanced. Each error bar covers more than $95 \%$ of the data set, with the remainder falling beyond the upper bound. The positive biases are greater in magnitude but smaller in quantity (only $37 \%$ are positive), resulting in the near-zero mean values.

To be useful, Fig. 5 is transformed to a relation between the standard deviation of biases and nondimensionalized particle velocity (Fig. 6). Given the value of $\bar{V}_{O} / V_{L}$, the possible biases in the velocity estimates can be immediately evaluated. Given the tolerance of biases and a known value of $\bar{V}_{O}$, the threshold velocity $V_{L}$ can be determined and used as the criterion for selecting a proper range of acoustic imaging. We present herein a first attempt to explore the potential of applying an acoustic camera in PTV, aiming to call for further research on this challenging subject.

${ }^{1}$ R. J. Adrian, Annu. Rev. Fluid Mech. 23, 261 (1991).

${ }^{2}$ R. N. Kieft, K. Schreel, G. van der Plas, and C. Rindt, Exp. Fluids 33, 603 (2002).

${ }^{3}$ K. Kim, N. Neretti, and N. Intrator, IEE Proc., Radar Sonar Navig. 152, 263 (2005).

${ }^{4}$ E. O. Belcher, Sea Technol. 47, 27 (2006).

${ }^{5}$ R. C. Gonzalez, R. E. Woods, and S. L. Eddins, Digital Image Processing Using MATLAB (Prentice-Hall, Upper Saddle River, NJ, 2004).

${ }^{6}$ B. Jähne, Digital Image Processing (Springer, Berlin, 2005).

${ }^{7}$ H. Capart, D. L. Young, and Y. Zech, Exp. Fluids 32, 121 (2002).

${ }^{8}$ D. Douxchamps, D. Devriendt, H. Capart, C. Craeye, B. Macq, and Y. Zech, Exp. Fluids 39, 535 (2005). 